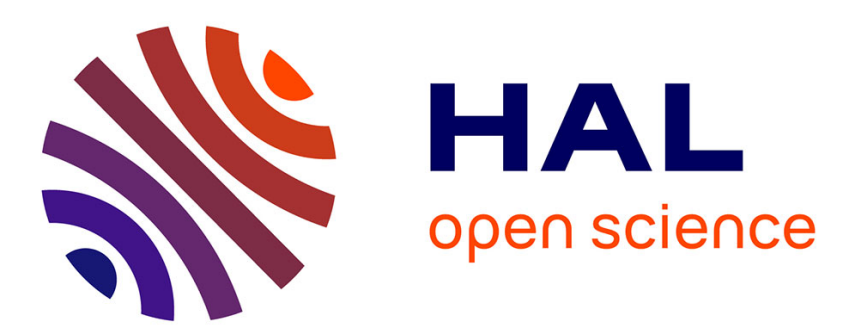

\title{
An experimental Comparison of State Observers for the Control of a Parallel Manipulator Without Velocity Measurements
}

Guilherme Sartori Natal, Ahmed Chemori, François Pierrot, Olivier Company

\section{- To cite this version:}

Guilherme Sartori Natal, Ahmed Chemori, François Pierrot, Olivier Company. An experimental Comparison of State Observers for the Control of a Parallel Manipulator Without Velocity Measurements. IROS: Intelligent Robots and Systems, Oct 2010, Taipei, Taiwan. pp.2301-2306, 10.1109/IROS.2010.5650400 . lirmm-00545491

\section{HAL Id: lirmm-00545491 https://hal-lirmm.ccsd.cnrs.fr/lirmm-00545491}

Submitted on 10 Sep 2019

HAL is a multi-disciplinary open access archive for the deposit and dissemination of scientific research documents, whether they are published or not. The documents may come from teaching and research institutions in France or abroad, or from public or private research centers.
L'archive ouverte pluridisciplinaire HAL, est destinée au dépôt et à la diffusion de documents scientifiques de niveau recherche, publiés ou non, émanant des établissements d'enseignement et de recherche français ou étrangers, des laboratoires publics ou privés. 


\title{
An Experimental Comparison of State Observers for the Control of a Parallel Manipulator Without Velocity Measurements
}

\author{
G. Sartori Natal and A. Chemori and F. Pierrot and O. Company
}

\begin{abstract}
This paper deals with the problem of unavailability of velocity measurements for the control of parallel manipulators for pick-and-place applications. As most controllers require the measurement of the joint positions as well as joint velocities, the latter need to be estimated. Three observers have been implemented while keeping the same nonlinear controller: a Lead-lag filter based observer, an Alpha-beta-gamma observer and a High-gain observer. The resulting performances obtained in Real-time experiments by each observer have been detailed and compared.
\end{abstract}

\section{INTRODUCTION}

Over the years, mechanical systems have served as an interesting benchmark for the design and validation of novel nonlinear control strategies. One problem that has attracted a good deal of interest is the output feedback control of mechanical systems. This problem is of practical importance since many commercially-available mechanical systems are not commonly equipped with velocity sensors (e.g., industrial robots) [1], which is our case with the two degree-of-freedom (dof) parallel robot Par2 [2], that can reach accelerations above 40G. The main reasons for the absence of velocity sensors on these mechanical systems are: (i) Signals delivered by tachometers might be contaminated with noise; (ii) Increased costs when more sensors are used in the plant [3].

It is well known that in most control algorithms it is assumed that the joint velocities are available. For this reason, they need to be calculated/estimated. The easiest way to compute the joint velocities consists in a numerical derivative of the measured joint positions. However, if the measured positions are noisy or do not have a good enough resolution, this technique will amplify the noise/quantization effect. An estimation of the joint velocities by means of observer-based techniques should then be considered.

The choice of the estimation mechanism is strongly influenced by the existence of uncertainty in the system model. Whereas model-based observers are usually restricted to cases where the model is exactly known, filters can provide model-independent means of estimating velocity [1].

Model-based schemes were presented in [4], where an exact knowledge based observer-controller was designed, which yielded a semi-global asymptotic tracking result, while in [5] and [6] the controlled system yielded semi-global exponential link position tracking. Reduced order observers

This work is supported by ANR Objectif 100G: a French National Project on Parallel Robots.

G. Sartori Natal, A. Chemori, F. Pierrot and O. Company are with LIRMM, Univ. Montpellier 2 - CNRS, 161 rue Ada, 34392 Montpellier, France sartorinatalelirmm. fr were designed for the case of exact knowledge-based controller [7] and adaptive controller [8]. For the case where the system was considered uncertain, variable structure modelbased observers for the design of adaptive [9] and robust [10] controllers were proposed. For more details about the control of PKMs, the user is referred to [11].

Filter-based control schemes were designed in [12], [13], [14], [15]. In [12], a simple first order filter was proposed to estimate the velocity for the force-position regulation of robotic manipulators through a $P I^{2} D$ controller. A filter on the position signal was used to create a velocity signal which was used to design a robust controller with feedforward terms with fixed parameter estimates and a linear feedback term was proposed in [14]. In [13], a filter-based observer was used with an adaptive output feedback controller which yielded semi-global asymptotic link position tracking. In [15], a filter was implemented to obtain the velocity error in an adaptive controller which was shown to guarantee global asymptotic trajectory tracking. A detailed comparison between three different methods of adaptive output feedback control for robotic manipulators (the first one with a Highgain observer (HGO), the second one with a linear secondorder observer and the third one with an Extended Kalman Filter (EKF)) was presented in [16].

While in [11] an experimental comparison between two controllers was made using the same velocity estimator (HGO), in the present work three velocity estimators have been implemented experimentally on Par2 parallel manipulator with the Dual Mode (DM) controller [17]: The Leadlag (LL) [18] based observer, the Alpha-beta-gamma (ABG) observer [19] and the High-gain observer [20]. The control performance obtained with each observer will be detailed and compared. It is important to emphasize that the parameters of the controller were not changed, as the main objective of this study is to evaluate the performance improvements obtained by using different observers with the same controller.

This paper is organized as follows. Section II introduces briefly the proposed control algorithm. In section III, the implemented observers are described. In section IV, the application is described. Section V is devoted to the experimental results, being the performances of the proposed observers compared and commented. In section VI, a conclusion about the current results is made.

\section{PROPOSED CONTROL SCHEME: A NONLINEAR DUAL MODE CONTROLER}

The control method proposed in this work is a nonlinear Dual Mode controller [17] (originally referred as 'binary'), 
derived from the nonlinear adaptive controller proposed in [21], with the addition of the projection on the law of the parametric adaptation. It consists basically in the utilization of a high adaptation gain together with a projection of the estimated parameters. Then, to large tracking errors in the transitory stage, the controller behaves approximately as a sliding mode controller, generating an exponential convergence to a residual domain arbitrarily small, and to smaller errors, it behaves as a parametric adaptation law. Other important advantages of the adaptation law in dual mode with respect to other adaptive controllers or known robust control algorithms are the generation of continuous control signals, the improvement of the robustness of the system and the limitation of the values of the estimated parameters thanks to the projection, which has the effect of reducing the effective gain of the controller when the tracking error increases (reducing the sensitivity to measuring noises).

This control law is given by:

$$
\tau=Y \hat{a}+K . s+\bar{d} \operatorname{Sat}(\alpha s)
$$

where $s=\dot{\tilde{q}}+\lambda \tilde{q}$, being $\tilde{q}=q_{d}-q, \dot{\tilde{q}}=\dot{q}_{d}-\dot{q}(q, \dot{q}$ and $\ddot{q}$ $\in \mathbb{R}^{n}$ are the vectors of positions, velocities and accelerations and $q_{d}, \dot{q}_{d}$ and $\ddot{q}_{d} \in \mathbb{R}^{n}$ are their desired trajectories, respectively); $\lambda, \bar{d}, \alpha$ and $K$ are positive constants, $\operatorname{Sat}(\alpha s)=$ $\frac{\alpha s}{\| \alpha s||+1}$ is a smooth and continuous saturation function with respect to its argument (with continuous partial derivatives and components limited to the interval $[-1,+1])$. The $m \times 1$ vector $\hat{a}$ represents an estimate of the unknown parameters of the system given by the vector $a$, and $Y(q, \dot{q}, \ddot{q}) \in \mathbb{R}^{n \times m}$ is the regressor vector (based on the dynamic model of the system). The reader is referred to [11] for more details about this controller.

According to equation (1), it is possible to notice that the proposed control scheme needs the measured positions and velocities. As mentioned before, it is assumed that the velocity measurements are not available, so an estimator must be proposed and implemented. In the present work, three estimators have been implemented and compared. They are described in the following section.

\section{IMPLEMENTED OBSERVERS}

To deal with the unavailability of velocity measurements, three estimation techniques have been implemented. They are presented in the sequel:

\section{A. LEAD-LAG BASED OBSERVER}

The Lead-lag filter [18] has the following modified structure:

$$
G_{l l}(p)=\frac{p}{\tau_{1} p+1}
$$

where $p$ is the Laplace variable. So, the smaller $\tau_{1}$ is chosen, the more accurate the velocity estimation will be, but the more noise the estimated velocity will have. Its basic idea is to approximately derivate the input signals (in our case, the joint positions), as shown in Eq. (3):

$$
\dot{\hat{q}}(t)=g_{l l}(t) * q(t)
$$

where $\dot{\hat{q}}(t)$ is the estimated joint velocity, $g_{l l}(t)$ is the inverse Laplace transform of $G_{l l}(p)$, i.e, $g_{l l}(t)=\mathscr{L}^{-1}\left[G_{l l}(p)\right]$ and the operator $*$ denotes the convolution between the two functions.

The main advantages of this method are its simplicity and computational efficiency, but its main drawbacks are:

1) If $\tau_{1}$ is given a big value, a delay will be generated and it will cause an inaccurate velocity estimation;

2) If $\tau_{1}$ is given a small value, there may be a considerable amplification of the noise/quantization effect.

Then, $\tau_{1}$ must be chosen such that a compromise between 1) and 2) is achieved.

\section{B. ALPHA-BETA-GAMMA OBSERVER}

The Alpha-beta (or Alpha-beta-gamma) observer [19] consists in a simplified form of Kalman Filter [22] for mechanical systems that have as states the positions and velocities. Its main idea consists in estimating the next step's states of the system according to the usual equations of kinematics and to correct these estimations through the multiplication of the positions' estimation errors by constant gains. The structure of this observer is detailed as follows:

- Estimation of the next step's positions and velocities:

$$
\left\{\begin{array}{c}
x_{\text {prev }}(k+1)=x_{\text {innov }}(k)+T v_{\text {innov }}(k)+\frac{1}{2} T^{2} a_{\text {innov }}(k) \\
v_{\text {prev }}(k+1)=v_{\text {innov }}(k)+T a_{\text {innov }}(k)
\end{array}\right.
$$

where $x_{\text {prev }}(k+1)$ and $v_{\text {prev }}(k+1)$ are the estimated positions and velocities for the next step, $x_{\text {innov }}(k), v_{\text {innov }}(k)$ and $a_{\text {innov }}(k)$ are respectively the updated positions, velocities and accelerations obtained through a correction based on the estimation error of the position, detailed as follows,

- Update of the estimated positions and velocities (also called innovation) based on the position measurement:

$$
\left\{\begin{array}{c}
x_{\text {innov }}(k)=x_{\text {prev }}(k)+\alpha\left(x_{\text {meas }}(k)-x_{\text {prev }}(k)\right) \\
v_{\text {innov }}(k)=v_{\text {prev }}(k)+\frac{\beta}{T}\left(x_{\text {meas }}(k)-x_{\text {prev }}(k)\right) \\
a_{\text {innov }}(k)=\frac{\gamma}{T^{2}}\left(x_{\text {meas }}(k)-x_{\text {prev }}(k)\right)
\end{array}\right.
$$

being $\alpha, \beta$ and $\gamma$ the update gains, $x_{\text {meas }}$ the measured positions and $T$ the sampling time.

In our case, the initial joint positions are known and the initial joint velocities are equal to zero, since the robot starts its movements from rest. So, $x_{\text {prev }}(1)$ and $v_{\text {prev }}(1)$ are initialized with these values, respectively. On the next step, based on the new measured joint positions, there is the innovation process, in which $x_{\text {innov }}, v_{\text {innov }}$ and $a_{\text {innov }}$ are computed and then used to compute the new estimated states of the system ( $x_{\text {prev }}$ and $v_{\text {prev }}$ ) for the next step.

As the Lead-lag based observer, this observer has also the advantage of being very computationally efficient and simple to implement, but it can provide a better performance and robustness (cf. experimental results in section V). One of its main disadvantages is that its gains must be chosen such that a considerable amplification of the noises/quantization effect 
is avoided, but even with less aggressive gains, fairly decent performances can be achieved.

\section{HIGH-GAIN OBSERVER}

The High-gain observer (HGO) is a model-based observer that was proposed in [20]. The main characteristic of this observer is that it takes into account the model of the system, so it can generate a very good performance if a precise model is available. However, even if this is not the case, this observer is robust because at each step a correction of the estimated states is made according to the comparison between the estimated positions and the measured ones. Its description is given as follows:

$\left\{\begin{array}{l}\dot{\hat{x}}_{1}=\hat{x}_{2}+\frac{1}{\varepsilon} \alpha_{H G O 1}\left(x_{1}-\hat{x}_{1}\right) \\ \hat{\hat{x}}_{2}=\frac{1}{\varepsilon^{2}} \alpha_{H G O 2}\left(x_{1}-\hat{x}_{1}\right)-\ddot{q}_{d}+\hat{F}\left(\hat{x}, q_{d}, \dot{q}_{d}\right)+\hat{H}\left(\hat{x}_{1}, q_{d}\right) \tau^{s}\end{array}\right.$

where

- $x_{1}$ represents the position error $\left(x_{1}=\tilde{q}\right)$ and $x_{2}$, its first derivative $\left(x_{2}=\dot{\tilde{q}}\right)$,

- $\hat{x}=\left[\begin{array}{ll}\hat{x}_{1} & \hat{x}_{2}\end{array}\right]^{T}$ represents the vector of estimated states,

- $\varepsilon, \alpha_{H G O 1}$ and $\alpha_{H G O 2}$ are positive gains,

- $\hat{F}\left(\hat{x}, q_{d}, q_{d}\right)$ and $\hat{H}\left(\hat{x}_{1}, q_{d}\right)$ are terms that depend on the dynamic model of the system,

- $\tau^{s}$ is the saturated torque (to avoid the 'peaking phenomena').

Its main characteristics are:

1) It is robust to uncertainties on the model of the system, although a precise model is needed in order to obtain better performances;

2) It is more complex (computationally and also concerning its implementation) than the two previously described observers;

3) If the gain is selected high enough, it becomes very sensitive to the noise in the position measurement.

\section{APPLICATION: PAR2 PARALLEL MANIPULATOR}

The three proposed observers, complied with the proposed nonlinear controller, were applied on the Par2 parallel manipulator illustrated in Fig. 1. Its main characteristics are:

- the platform (b) is a rigid body,

- only the two inner arms (3) are actuated,

- the two other arms (4) are linked to the frame (1) through passive revolute joints,

- inner arms (3) and (4) are connected to (6) with pairs of rods (5) mounted on ball joints (7),

- the rotations of the arms (4) are coupled in order to guarantee planar motions along $\mathrm{x}$ and $\mathrm{z}$ axes.

For more details about the prototype Par2, the reader is referred to [2].

\section{REAL-TIME EXPERIMENTAL RESULTS}

The objective of this section is to present and discuss realtime experimental results obtained by the application of the three proposed observers described in section III associated
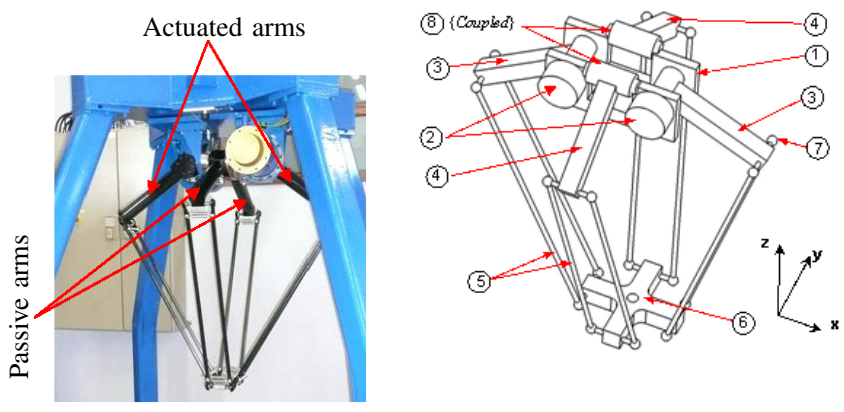

Fig. 1. The two-dof parallel manipulator Par2: view of the robot (left), schematic view of its mechanical structure (right)

with the control scheme described in section II to the Par2 parallel manipulator described in section IV. The Real-time experimental setup is displayed in Fig. 2.

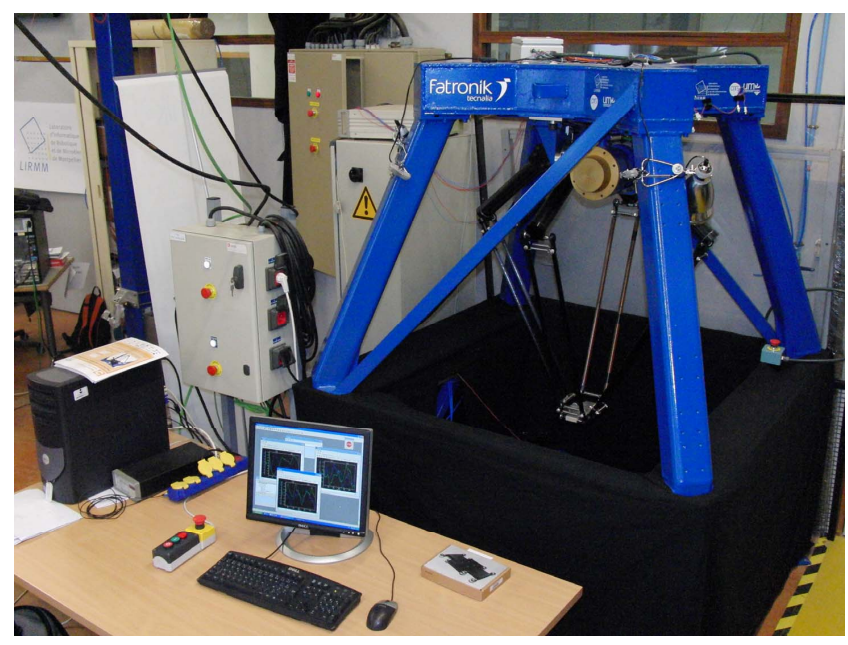

Fig. 2. Par2 parallel manipulator experimental setup

The platform of the robot evolves in the $X O Z$ plane (cf. figure 1). The desired Cartesian trajectory to be tracked by Par2 is a usual 'pick-and-place' trajectory (which has an S-curve profile, being the reader referred to [23] for more details). Its parameters are described in table I.

The parameters of the proposed control approach are summarized in table II and the parameters of the three proposed observers are described in table III. These parameters were tuned such that each observer could provide its best performance.

TABLE I

PARAMETERS OF THE CARTESIAN REFERENCE TRAJECTORY

\begin{tabular}{|c|l|c|}
\hline Parameter & Description & Value \\
\hline$\left(x_{d_{i}}, z_{d_{i}}\right)$ & $\begin{array}{l}\text { Initial desired Cartesian position } \\
\text { in the plane } X O Z\end{array}$ & $(-0.35 \mathrm{~m},-0.95 \mathrm{~m})$ \\
\hline$\left(x_{d_{f}}, z_{d_{f}}\right)$ & $\begin{array}{l}\text { Final desired Cartesian position } \\
\text { in the plane } X O Z\end{array}$ & $(0.35 \mathrm{~m},-0.95 \mathrm{~m})$ \\
\hline$\dot{x}_{d}^{\max }$ & Maximum Cartesian velocity & $7 \mathrm{~m} / \mathrm{s}$ \\
\hline$\ddot{x}_{d}^{\max }$ & Maximum Cartesian acceleration & $15 \mathrm{G}$ \\
\hline
\end{tabular}

The proposed experimental scenario deals with the control 
TABLE II

PARAMETERS OF THE CONTROL APPROACH

\begin{tabular}{|c||c|}
\hline Parameter & Description \\
\hline$K_{p}=94.5$ & Proportional gain \\
\hline$K_{d}=2.1$ & Derivative gain \\
\hline$\lambda=25$ & Positive constant \\
\hline$K=2 I$ & Matrix gain \\
\hline $\bar{d}=2.5$ & Smooth variable structure gain \\
\hline$\alpha=0.05$ & Smooth variable structure slope \\
\hline$M_{\theta}=0.25$ & Max. adaptative parameters' error \\
\hline$\gamma=0.3345$ & Adaptive gain \\
\hline$T_{s}=0.0005$ & Sampling time (s) \\
\hline$n=3$ & Number of cycles \\
\hline
\end{tabular}

TABLE III

PARAMETERS OF THE PROPOSED OBSERVERS

\begin{tabular}{|c||c|}
\hline$\tau_{1}=0.001$ & LL time constant \\
\hline$\varepsilon=0.002, \alpha_{H G O 1}=\alpha_{H G O 2}=1$ & HGO gains \\
\hline$\alpha_{a b g}=0.95, \beta_{a b g}=\gamma_{a b g}=0.5$ & ABG gains \\
\hline
\end{tabular}

of the Par2 parallel manipulator for one cycle of a pickand-place trajectory, that is, the robot's platform has to go (as illustrated in Fig. 3) from the desired 'pick' Cartesian position $\left(x_{d_{i}}, z_{d_{i}}\right)$ to the desired 'place' Cartesian position $\left(x_{d_{f}}, z_{d_{f}}\right)$ and then return to the initial one $\left(x_{d_{i}}, z_{d_{i}}\right)$. The corresponding Cartesian reference trajectory and the illustration of the robots movements are shown in Fig. 3.

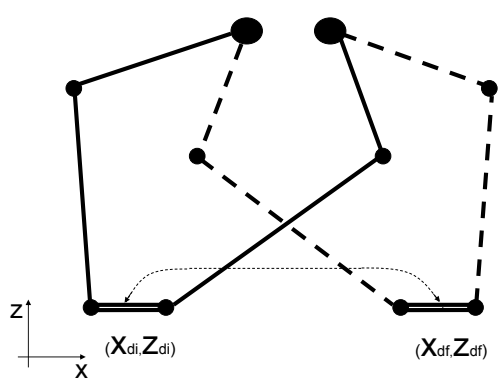

Fig. 3. Illustration of the robot's movements

The desired joint trajectory denoted by $q_{d}(t)$ is supposed uniformly bounded, twice continuously differentiable with its two first derivatives $\dot{q}_{d}(t)$ and $\ddot{q}_{d}(t)$ also uniformly bounded.

The trajectory tracking obtained by the DM controller with the three proposed observers for $15 \mathrm{G}$ of maximum acceleration is shown in Figs. 4 and 5, and the corresponding tracking errors are shown in Fig. 6. The velocity tracking is presented in Fig. 7 and the estimated velocity errors are presented in Figs. 8 and 9. The control inputs are shown in Figs. 10 and 11. The real-time implementation of these observers was made with a sampling time of $0.5 \mathrm{msec}$.

It is worth to notice that the experimental testbed does not include a sensor to measure the Cartesian position of the platform [2]. In addition, because of the high frequency rate (which requires a small computing time), other methods such as vision-based control have not been implemented.

By analyzing figures 5 and 6 , it is possible to notice that the best tracking performance was obtained with the $\mathrm{ABG}$
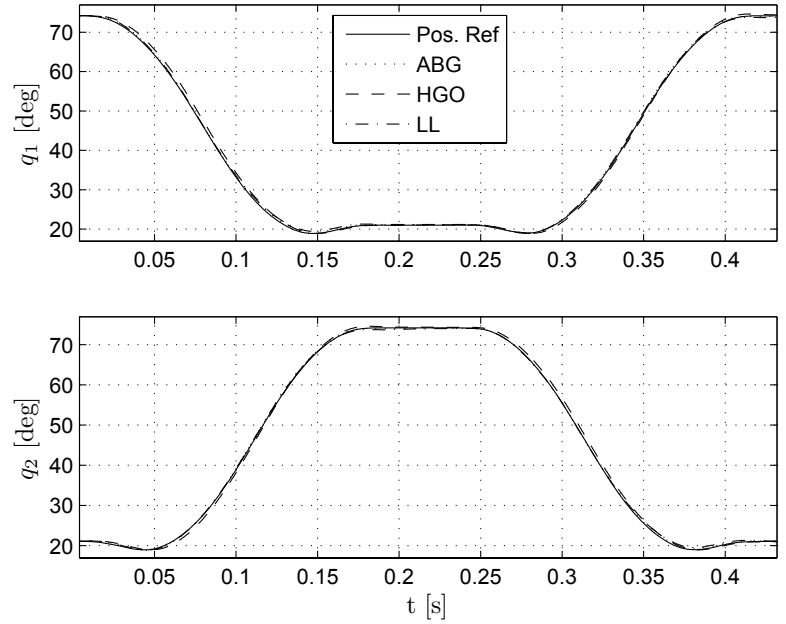

Fig. 4. One cycle pick-and-place trajectory trackings for $15 \mathrm{G}$ obtained by using the ABG (dots), the HGO (dashed) and the LL (dash-dots) observers
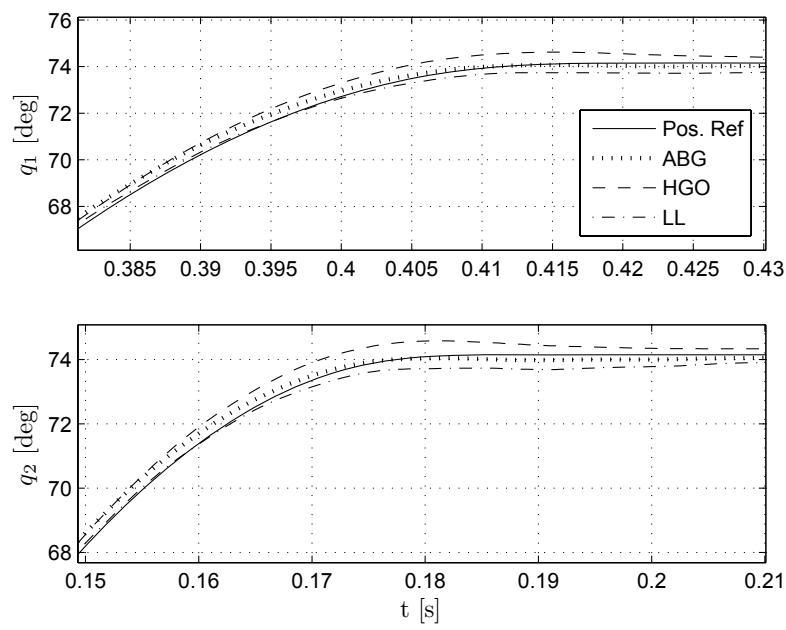

Fig. 5. Zoom on the trajectory tracking around stop points for $15 \mathrm{G}$

observer. With this observer, the tracking errors were kept inside the limits of $\left[-0.2^{\circ}, 0.55^{\circ}\right]$ during all the trajectories, while the LL observer kept them inside the limits of $\left[-0.5^{\circ}, 0.7^{\circ}\right]$ and the HGO kept them inside the limits of $\left[-0.6^{\circ}, 1.2^{\circ}\right]$ (peak-to-peak improvement of approximately $37.5 \%$ and $58.3 \%$, respectively). For further analysis, their Root Mean Square Error (RMSE) have also been calculated. With the ABG observer, a RMSE of $0.212^{\circ}$ was obtained, while $0.291^{\circ}$ and $0.518^{\circ}$ were obtained with the LL and the HGO, respectively. This means an improvement of $27.15 \%$ in comparison with the LL observer and approximately $50 \%$ in comparison with the HGO.

As for the main reasons why the Alpha-beta-gamma observer was able to generate a better tracking performance than the Lead-lag based observer and the High-gain observer, one can mention that:

1) For the implementation of the HGO, important simplifications on the model of the system were made (when 

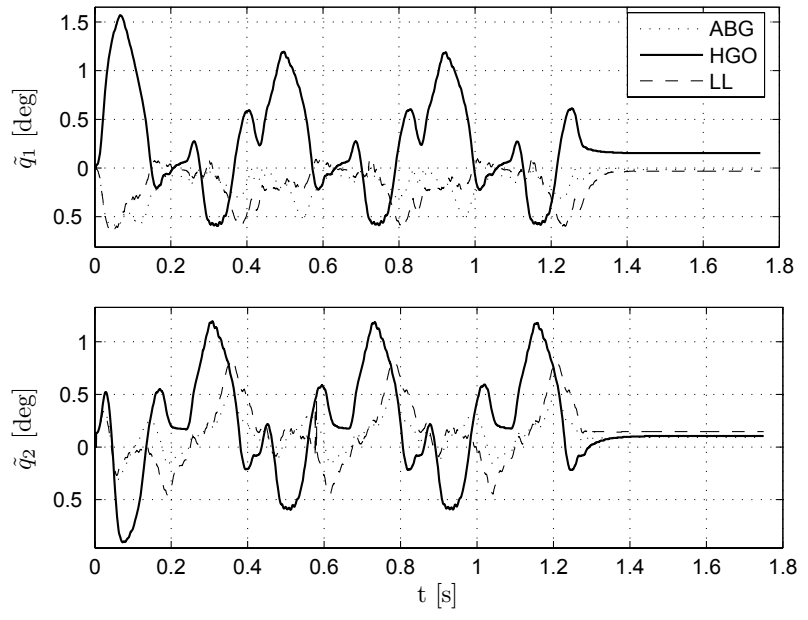

Fig. 6. Tracking errors for $15 \mathrm{G}$ obtained by using the HGO (solid), for the ABG (dots) and for the LL (dashed)
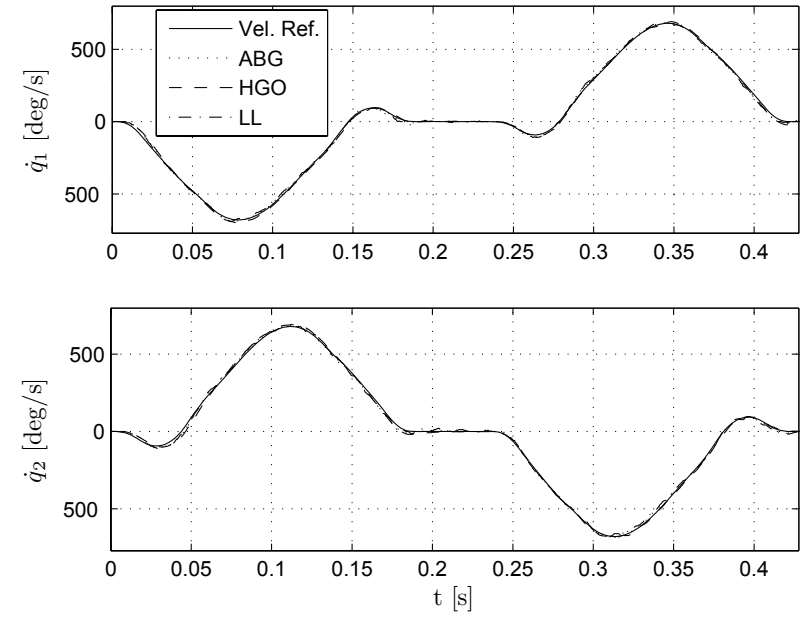

Fig. 7. One cycle velocity trackings obtained by using the ABG (dots), the HGO (dashed) and the LL (dash-dots) observers

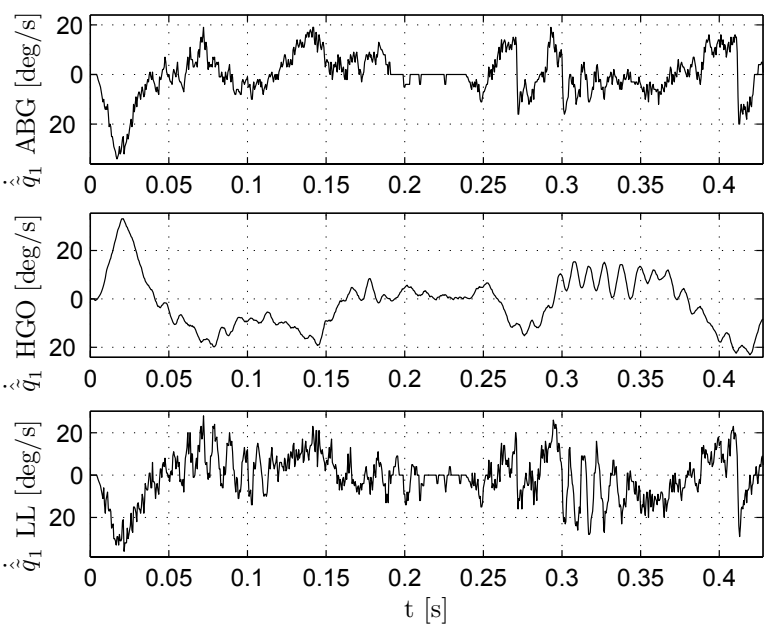

Fig. 8. One cycle estimated velocity errors (peak errors inside limit of 5\% of the velocity amplitudes) obtained by using each observer (Motor 1)

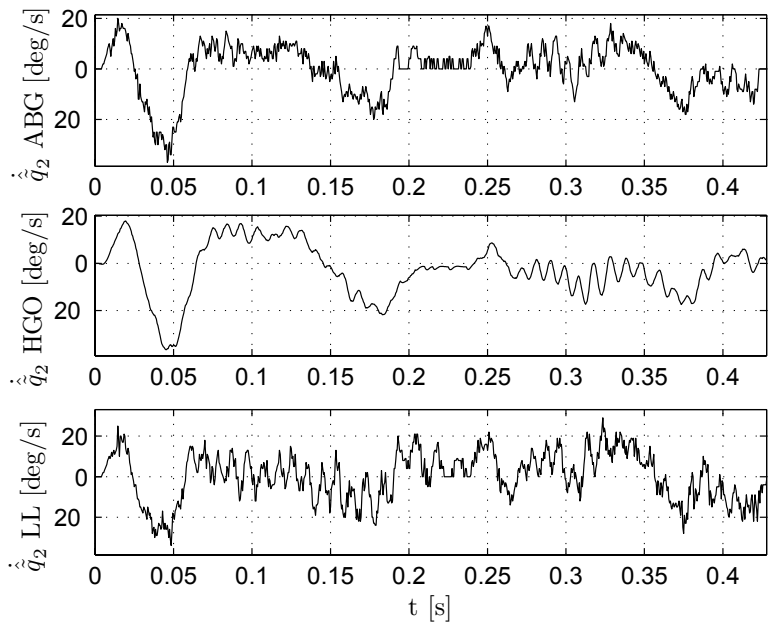

Fig. 9. One cycle estimated velocity errors (peak errors inside limit of 5\% of the velocity amplitudes) obtained by using each observer (Motor 2)

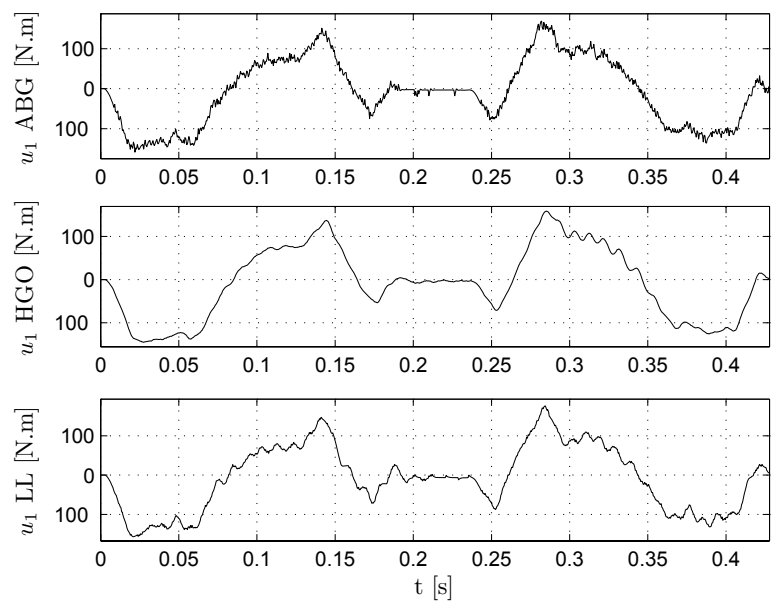

Fig. 10. One cycle torques obtained by using each observer (Motor 1)

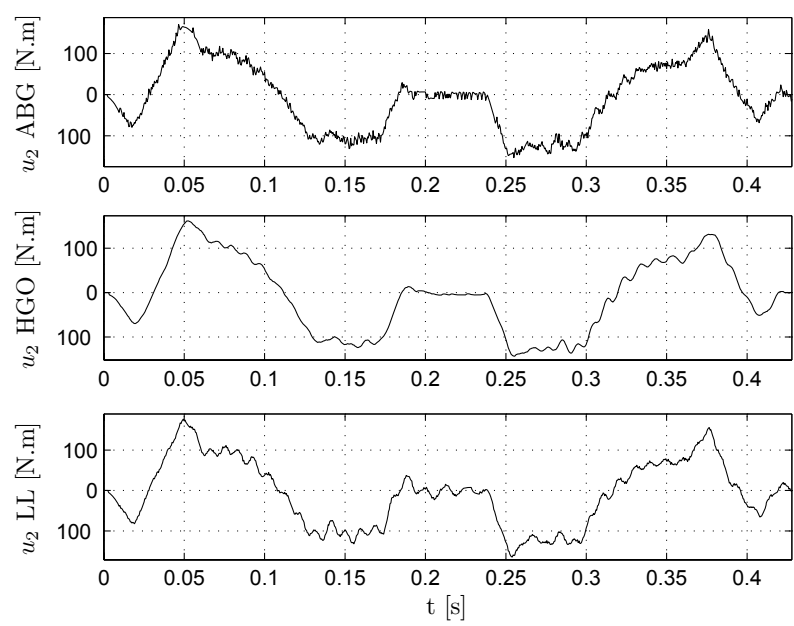

Fig. 11. One cycle torques obtained by using each observer (Motor 2) 
neglecting the mass of the platform) such that it would be possible to represent it on the Lagrangian form. As this observer is model-dependent, this may have caused a considerable loss of performance;

2) The $A B G$ observer is naturally more performant than the LL based observer because the last one consists only in a transfer function that will generate an approximate value of the velocity, while the ABG observer not only estimates the velocity but also corrects its estimation at each step time according to the estimation of the position error.

From figure 7 , it is difficult to distinguish the curves of reference velocities from those of the estimated ones. It is only possible to adequately analyze their estimation performances through the estimated velocity errors in Fig. 8 (for motor 1) and figure 9 (for motor 2). These figures show that the velocity estimation of the $\mathrm{ABG}$ observer was the one that, in average, kept the smaller errors for the longest time during the trajectories, while the velocity estimation of the HGO was the one that kept the smaller errors for the shortest time. It is also possible to notice that the $A B G$ observer generated the most noisy velocity signals, while the LL generated smaller noises but bigger oscillations and the HGO generated nearly no noise and small oscillations.

As for the control signals (figure 10 for motor 1 and figure 11 for motor 2), a similar conclusion can be made. The ABG observer generated the most noisy control signals with small vibrations, the HGO generated the smoothest signals with small vibrations and the LL observer generated the signals with the biggest oscilations. The control signals generated by the three observers showed roughly similar amplitudes and remained inside the admissible limits of each actuator (500 N.m) by a large margin.

The performance details of the three observers are summarized on table IV.

TABLE IV

PERFORMANCE COMPARISON BETWEEN THE OBSERVERS

\begin{tabular}{|c||c||c||c|}
\hline Performance & ABG & LL & HGO \\
\hline Error peaks & {$\left[-0.2^{\circ}, 0.55^{\circ}\right]$} & {$\left[-0.5^{\circ}, 0.7^{\circ}\right]$} & {$\left[-0.6^{\circ}, 1.2^{\circ}\right]$} \\
\hline RMSE & $0.212^{\circ}$ & $0.291^{\circ}$ & $0.518^{\circ}$ \\
\hline \multirow{2}{*}{ Control signals } & More noisy & More oscillating & Smoother \\
\cline { 2 - 4 } & \multicolumn{2}{|c|}{ Roughly similar amplitude values } \\
\hline
\end{tabular}

\section{CONCLUSIONS}

This paper deals with the problem of unavailability of velocity measurements for the control of parallel manipulators. Three state observers have been proposed and implemented, namely the Alpha-beta-gamma (ABG) observer, the Highgain observer (HGO) and a Lead-lag (LL) based observer. A detailed comparison between the trajectory tracking performances obtained by these observers associated with a nonlinear Dual Mode (DM) controller for a parallel manipulator (Par2) has been presented, for a maximum acceleration of 15G. By analyzing the obtained results, it was possible to notice that all observers generated a good estimation of the velocities, which made it possible for the DM controller to have a good tracking performance in all cases. However, the ABG observer generated the best tracking performance.

\section{REFERENCES}

[1] B. Xian, M. S. Queiroz, D. M. Dawson, and M. L. McIntyre, "A discontinuous output feedback controller and velocity observer for nonlinear mechanical systems," Automatica, vol. 40, pp. 695-700, 2004.

[2] F. Pierrot, C. Baradat, V. Nabat, O. Company, S. Krut, and M. Gouttefarde, "Above 40g acceleration for pick-and-place with a new 2-dof pkm," in Proc. IEEE Conf. Robotics Automat., (Kobe, Japan), 2009.

[3] M. A. Arteaga, "Robot control and parameter estimation with only joint position measurements," Automatica, vol. 39, pp. 67-73, 2003.

[4] S. Nicosia and P. Tomei, "Robot control using only joint position measurements," IEEE Trans. on Automat. Contr., vol. 15, no. 9, pp. 1058-1061, 1990.

[5] H. Berghuis and H. Nijmeijer, "A passivity approach to controllerobserver design for robots," IEEE Trans. on Robotics Automat., vol. 9, pp. 740-754, 1993.

[6] S. Y. Lim, D. M. Dawson, and K. Anderson, "Re-examining the Nicosia-Tomei robot observer-controller from a backstepping perspective," IEEE Trans. Contr. Syst. Technol., vol. 4, no. 3, pp. 304-310, 1996.

[7] M. Erlic and W. Lu, "Manipulator control with en exponentially stable velocity observer," ACC, pp. 1241-1242, 1992.

[8] M. Erlic and W. Lu, "A reduced-order adaptive velocity observer for manipulator control," CRA, pp. 328-333, 1993.

[9] C. C. de Wit, N. Fixot, and K. J. Aström, "Trajectory tracking in robot manipulators via nonlinear estimated state feedback," IEEE Trans. on Robotics Automat., vol. 18, no. 1, pp. 138-144, 1992.

[10] C. C. de Wit, N. Fixot, and K. J. Aström, "Robot control via robust estimated state feedback," IEEE Trans. on Automat. Contr., vol. 36, pp. 1497-1501, 1991.

[11] G. Sartori-Natal, A. Chemori, F. Pierrot, and O. Company, "Nonlinear dual mode adaptive control of par2: a 2-dof planar parallel manipulator, with real-time experiments," Proc. IEEE/RSJ Int. Conf. Intel. Robotics Systems., pp. 2114-2119, 2009.

[12] A. Loria and R. Ortega, "Force/position regulation for robot manipulators with unmeasured velocities and uncertain gravity," Automatica, vol. 32, no. 6, pp. 939-943, 1996.

[13] K. Kaneko and R. Horowitz, "Repetitive and adaptive control of robot manipulators with velocity estimation," IEEE Trans. on Robotics Automat., vol. 13, no. 2, pp. 204-217, 1997.

[14] J. Yuan and Y. Stepanenko, "Robust control of robotic manipulators without velocity measurements," Int. J. Rob. Nonl. Contr., vol. 1, pp. 203-213, 1991.

[15] E. Zergeroglu, D. M. Dawson, M. S. de Queiroz, and M. Krstic, "On global output feedback tracking control of robot manipulators," Proc. 39th. IEEE Conf. Decision Contr., pp. 5073-5078, 2000.

[16] J. M. Daly and H. M. Schwartz, "Experimental results for output feedback adaptive robot control," Robotica, vol. 24, pp. 727-738, 2006.

[17] L. Hsu and R. R. Costa, "B-mrac global exponential stability with a new model reference adaptive controller based on binary control theory," Contr. Theory Adv. Technol., vol. 10, no. 4, pp. 649-668, 2003.

[18] S. N. Norman, Control Systems Engineering. Wiley \& Sons, 4th Ed., 2004.

[19] R. Penoyer, “The alpha-beta filter," C User's Journal, vol. 11, no. 7, pp. 73-86, 1993.

[20] K. W. Lee and H. K. Khalil, "Adaptive output feedback control of robot manipulators using high-gain observer," Int. J. of Contr., vol. 67, no. 6, pp. 869-886, 1997.

[21] J. Slotine and W. Li, "Adaptive manipulator control: A case study," IEEE Trans. on Automat. Contr., vol. 33, no. 11, pp. 995-1003, 1988.

[22] R. E. Kalman, "A new approach to linear filtering and prediction problems," Transactions of the ASME - Journal of Basic Engineering, pp. 35-45, 1960.

[23] E. Red, "A dynamic optimal trajectory generator for cartesian path following," Robotica, vol. 18, pp. 451-458, 2000. 